\title{
DYNAMIC CHARACTERIZATION OF A LINEAR VARIABLE-CAPACITANCE MICROMOTOR
}

\author{
Nima Ghalichechian ${ }^{1}$, Alireza Modafe ${ }^{1}$, Alex Frey ${ }^{1}$, Jeffery H. Lang ${ }^{2}$, and Reza Ghodssi ${ }^{1}$ \\ ${ }^{1}$ MEMS Sensors and Actuators Lab (MSAL) \\ Department of Electrical and Computer Engineering, Institute for Systems Research \\ University of Maryland, College Park, MD USA \\ ${ }^{2}$ Department of Electrical Engineering and Computer Science \\ Massachusetts Institute of Technology, Cambridge, MA USA \\ Email: ghodssi@eng.umd.edu
}

\begin{abstract}
We report, for the first time, the dynamic characterization of a 6-phase, bottom-drive, linear, variable-capacitance micromotor (B-LVCM) supported on microball bearings. The motion of the micromotor was captured using a high-speed video camera, tracked using an image processing software, and analyzed to obtain the instantaneous velocity $(11 \mathrm{~mm} / \mathrm{s})$, acceleration $\left(1.9 \mathrm{~m} / \mathrm{s}^{2}\right)$, and net force $(0.19 \mathrm{mN})$ (all in amplitude) from the position data. This characterization provides an understanding of the dynamic behavior of both the variable-capacitance micromachines and the microball bearings on which they are supported.
\end{abstract}

\section{INTRODUCTION}

Microball bearing technology in silicon provides a reliable and robust support mechanism for the rotor of micromotors and microgenerators. Design and fabrication of the first-generation device, a 3-phase B-LVCM supported on microball bearings, was demonstrated in our previous work [1]. The primary application of the B-LVCM (shown in Figure 1) is long-range, high-speed, linear micropositioning. More important, this device provides a platform to investigate the mechanical properties of roller bearings in MEMS. The initial testing of the linear micromotor was reported in [2]. The development of the linear micromotor is based on several studies on the motor core components. An in situ non-contact experimental system was developed to study the frictional behavior of the microballs in the microscale regime. Static and dynamic coefficient of friction of stainless steel microballs and the silicon grooves, fabricated by anisotropic silicon etching using potassium hydroxide $(\mathrm{KOH})$, were measured to be 0.01 and 0.007 , respectively [3], compared to 0.01-0.08 reported for silicon-silicon structures $[4,5]$. The dielectric constant and breakdown voltage of benzocyclobutene (BCB) low-k polymer, used as an electrical insulator for reducing parasitic losses, were measured and the effect of humidity on these properties was studied [6]. A fabrication process for integration of BCB film with wet etching of silicon using $\mathrm{KOH}$ was developed [7]. The electrical isolation of the electrodes from the silicon substrate in the previously fabricated device was achieved by a novel fabrication process (Embedded BCB in Silicon, or EBiS) to planarize isolated islands of low-k BCB polymer in silicon [8]. While a great deal of research has been conducted on the design and fabrication of micromachines, little work has been done on drive, control, test, and characterization of these machines. The dynamic characterization of the 6-phase micromotor including the measurement results of position, instantaneous velocity, acceleration, and the net force on the slider are presented in this paper.

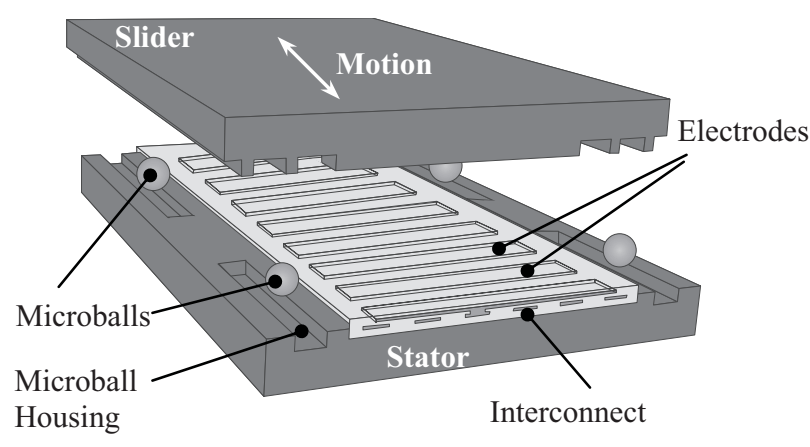

Figure 1: Schematic $3 D$ view of the bottom-drive, linear, variable-capacitance micromotor [1].

\section{DESIGN}

Figure 1 shows a 3D exploded view of the linear micromotor supported on microball bearings. The micromotor comprises three major components: stator, slider, and microballs. Unlike the (successfully tested) conventional VCMs that were side-drive [9], this micromotor has a bottom-drive design which increases the active area and force of the motor. The microball bearing design provides the mechanical support for maintaining a uniform air gap that was not possible in conventional (side-drive or top-drive) VCMs with center-pin or flange designs.

Two new technologies were implemented in the development of the linear micromotor: (1) microball bearing technology in silicon as a support mechanism and (2) BCB low-k polymer as an insulating layer $[\mathbf{1}, \mathbf{2}]$. The microball bearing technology results in stable, robust, and reliable mechanical support. The rolling microballs, sandwiched between the rotor and stator, results in less friction and wear than center-pin design and are more reliable and stable than gas-lubricated bearings. A conventional dielectric film of silicon dioxide $\left(\mathrm{SiO}_{2}\right)$ was replaced by a low-k $(\mathrm{k}=2.65) \mathrm{BCB}$ polymer. This results in a reduced parasitic capacitance and an increased efficiency of the motor. BCB also exhibits less residual stress than $\mathrm{SiO}_{2}$; therefore, the device has less curvature and the air gap is more uniform. The geometry specifications of the tested B-LVCM are shown in Table 1.

\section{FABRICATION}

The second-generation B-LVCM was fabricated with some modifications to the process of first-generation device. The fabrication process of the stator comprises the following steps: (1)

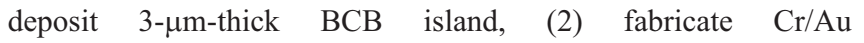


interconnections and pattern photo-BCB to open vias, (3) fabricate $\mathrm{Cr} / \mathrm{Au}$ electrodes and pattern photo-BCB passivation layer, and (4) pattern and etch trenches with deep reactive ion etching (DRIE) for housing the microballs. The slider fabrication process comprises a lithography step and a DRIE step to etch both trenches and poles. The yield of the $\mathrm{Cr} / \mathrm{Au}$ metallization process was increased by replacing the original lift-off process with an etch process. Figures 2 and 3 show the fabricated stator and slider of the 6-phase micromotor, respectively.

Table 1: Micromotor geometry specifications (electrodes refer to the stator and poles refer to the salient structures on the rotor).

\begin{tabular}{|c|c|}
\hline Parameter & Value \\
\hline Electrode/pole width, $\mu \mathrm{m}$ & $90 / 90$ \\
\hline Electrode/pole pitch, $\mu \mathrm{m}$ & $120 / 180$ \\
\hline Electrode/pole number & $84 / 36$ \\
\hline Air gap, $\mu \mathrm{m}$ & $26-34$ \\
\hline Microball diameter, $\mu \mathrm{m}$ & 284.5 \\
\hline Trench width, $\mu \mathrm{m}$ & 290 \\
\hline Microball material & $\begin{array}{c}\text { 440C stainless } \\
\text { steel }\end{array}$ \\
\hline
\end{tabular}

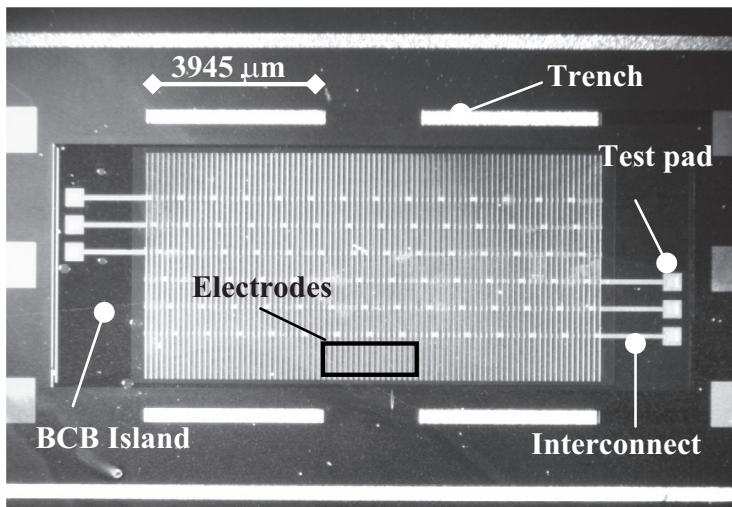

Figure 2: Optical micrograph (top-view) of the 6-phase stator comprises three BCB layers, two metal layers, and four trenches for housing microballs.

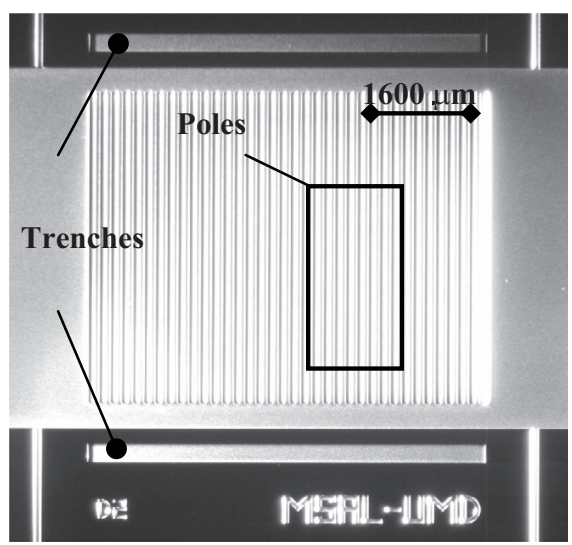

Figure 3: Optical micrograph (top-view) of the slider showing poles and trenches etched into silicon substrate using DRIE.

\section{TEST SETUP}

A new test-bed for the characterization of the micromotor was developed. Figure 4 shows the block diagram of the test

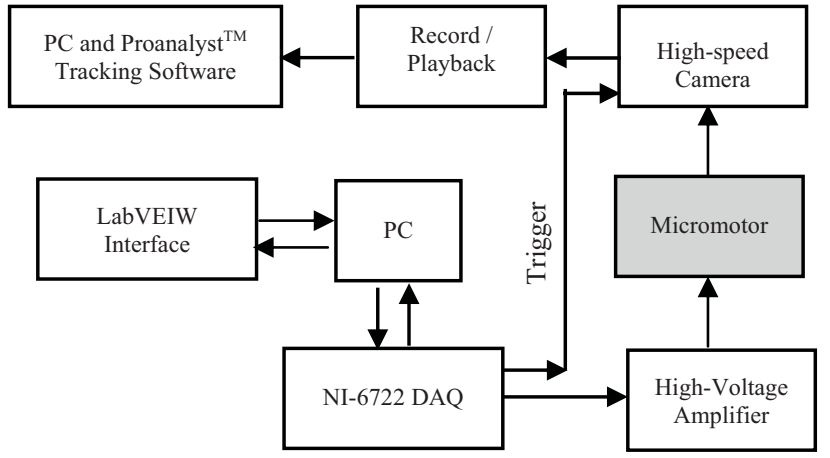

Figure 4: Block diagram of the micromotor test station.

station compromised of six-channel high-voltage actuation system, high-speed camera, and image processing division. Initially, the motion of the motor was captured using a 30-frame-per-second (fps) camera. The speed of a standard 30-fps camera was found to be low for capturing the transient response of the micromotor. Therefore, real-time measurement was performed using a lownoise, monochromatic, high-speed (635-1000 fps) video camera (MotionPro HS-3 from Redlake). This real-time measurement was preferred over conventional stroboscopic dynamometry techniques previously used for side-drive micromotors [10].

\section{RESULTS}

The micromotor is excited with six square-wave pulses. The first three phases are $120^{\circ}$ out of phase. The remaining three phases have the inverse amplitude of the first three. This excitation configuration prevents charge build-up on the slider. Figures 5 and 6 show the displacement of the slider measured for an excitation frequency $\left(f_{e}\right)$ of 10 and $20 \mathrm{~Hz}$, respectively, and acquisition rate of 30 -fps. Since the range of motion in this design, is limited to 3.4 $\mathrm{mm}$, the direction of motion is changed (forward and backward) with a frequency of $1.5 \mathrm{~Hz}$. This is done by switching the sequence of the phases. The saw-tooth behavior, shown in figures 5 and 6 , is a result of the change in the direction of the motion.

The average velocity $\left(V_{\text {avg }}\right)$ of the slider is given by

$$
V_{\text {avg }}=2 W f_{e}
$$

where $W$ is the width of an electrode or pole and $f_{e}$ is the frequency of excitation voltage. The average velocity can be obtained from

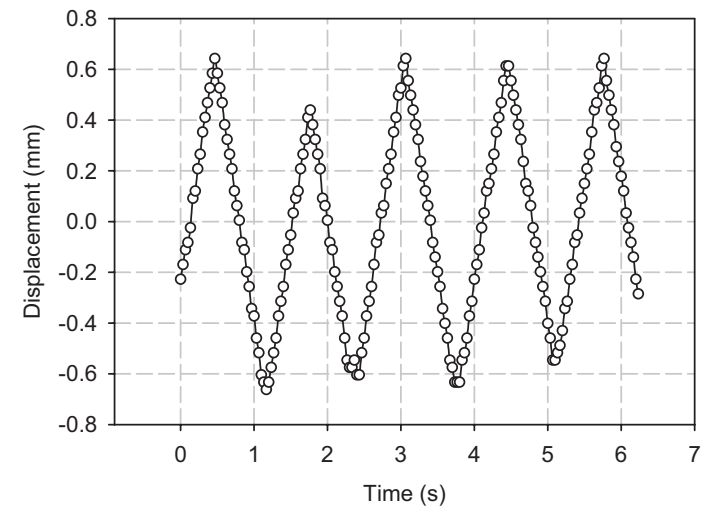

Figure 5: Displacement of the slider when excited with $120 \mathrm{~V}, 10 \mathrm{~Hz}$, six-phase, square pulses and measured using a 30-fps camera. The direction of the motion alternates with $f_{d}=1.5 \mathrm{~Hz}$. 
the slopes of the two graphs (Figures 5 and 6). Similar measurements were performed at $40 \mathrm{~Hz}$ and $80 \mathrm{~Hz}$ excitation. The results, summarized in Table 2, show a good agreement between predicted and measured values of $V_{\text {avg }}$ for $f_{e} \leq 60 \mathrm{~Hz}$.

The transient response of the micromotor to $120-\mathrm{V}$ square pulses was measured at $1000 \mathrm{fps}$ while the camera's capture sequence was synchronized to the excitation voltage. Figure 7 shows the acceleration of the slider from rest position to a quasisteady-state in about $20 \mathrm{~ms}$ (first region). After this period the machine continues with $V_{\text {avg }}$ (second region), however, the instantaneous acceleration is not zero and the velocity changes in a periodic fashion.

Figure 8 shows the oscillation of the rotor position, $X(t)$, in a 130-ms window captured at $635 \mathrm{fps}$. In order to study the transient response of the machine, the linear component of the displacement signal, $\left(V_{\text {avg }} \times t\right)$, was filtered from Figure 8 and the remaining component, $\left.\left(X(t)-V_{\text {avg }} \times t\right)\right)$, was plotted in Figure 9. A damped sinusoidal transient response of the rotor can be seen in this figure. The instantaneous velocity and acceleration were computed from the first and second derivatives of the fitted curve (Figure 9) and are shown in figures 10 and 11 , respectively. Figure 11 also demonstrates the instantaneous net force on a 0.1-gram slider (difference between lossless electromechanical and friction force) that is comparable to our models.

The micromotor was modeled as a mass-dashpot-spring system where $m$ is the mass of the rotor, $b$ is a dashpot coefficient representing the friction of microballs and silicon housing, and $k$ is the spring constant of the electrode-pole variable capacitors. Solving the $2^{\text {nd }}$ order differential equation of the system yields an oscillating function with decaying amplitude (underdamped solution). Thus, the dashpot coefficient (b) was extracted from the decay envelop of Figure 9 and found to be $6.25 \times 10^{-4} \mathrm{Kg} / \mathrm{s}$. This value can be used to characterize the tribological behavior of microball bearings in comparison to macroball bearings.

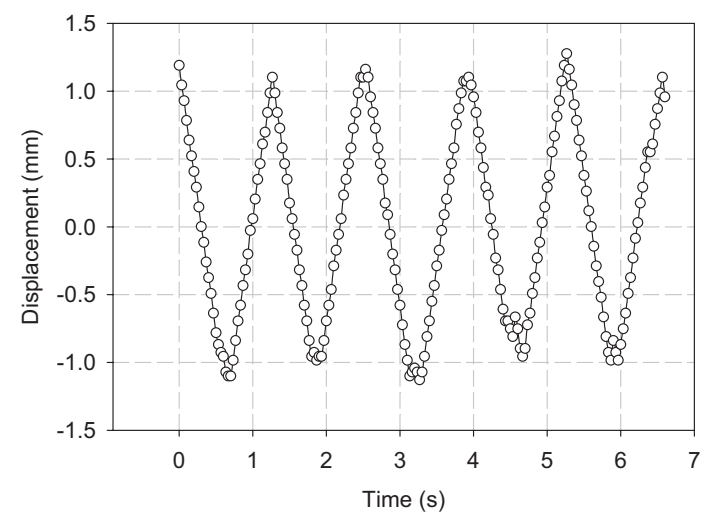

Figure 6: Displacement of the slider when excited by $120 \mathrm{~V}, 20 \mathrm{~Hz}$, six-phase, square pulses and measured using a 30-fps camera $\left(f_{d}=1.5 \mathrm{~Hz}\right)$.

Table 2: Comparison of predicted and measured average velocity of the micromotor.

\begin{tabular}{|c|c|c|}
\hline $\begin{array}{c}\text { Excitation } \\
\text { frequency } \\
\text { (Hz) }\end{array}$ & $\begin{array}{c}\text { Predicted } \\
\text { average } \\
\text { velocity } \\
\text { (mm/s) }\end{array}$ & $\begin{array}{c}\text { Measured } \\
\text { average } \\
\text { velocity } \\
\text { (mm/s) }\end{array}$ \\
\hline $\mathbf{1 0}$ & 1.80 & $1.94 \pm 0.02$ \\
\hline $\mathbf{2 0}$ & 3.60 & $3.98 \pm 0.02$ \\
\hline $\mathbf{4 0}$ & 7.20 & $7.37 \pm 0.12$ \\
\hline $\mathbf{8 0}$ & 14.40 & $7.21-11.10$ \\
\hline
\end{tabular}

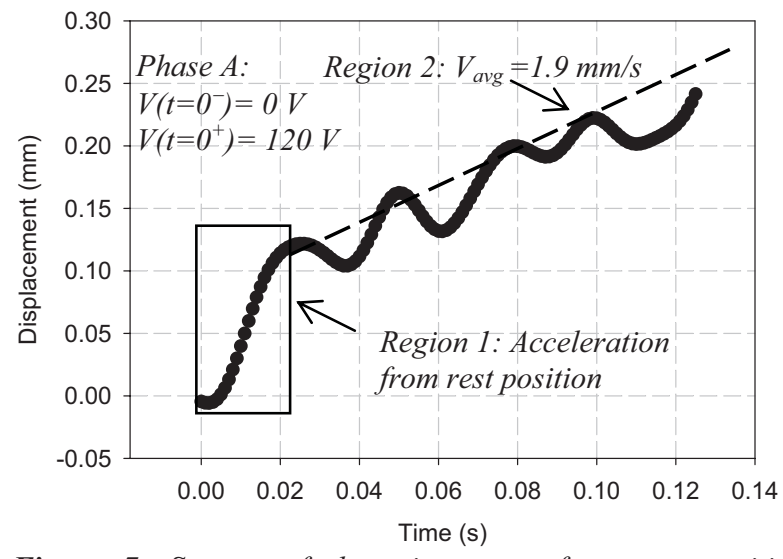

Figure 7: Startup of the micromotor from rest position captured with a 1000-fps camera showing two regions of operation: (1) acceleration from rest position and (2) quasisteady-state operation with average velocity of $V_{\text {avg }}$ and local oscillations.

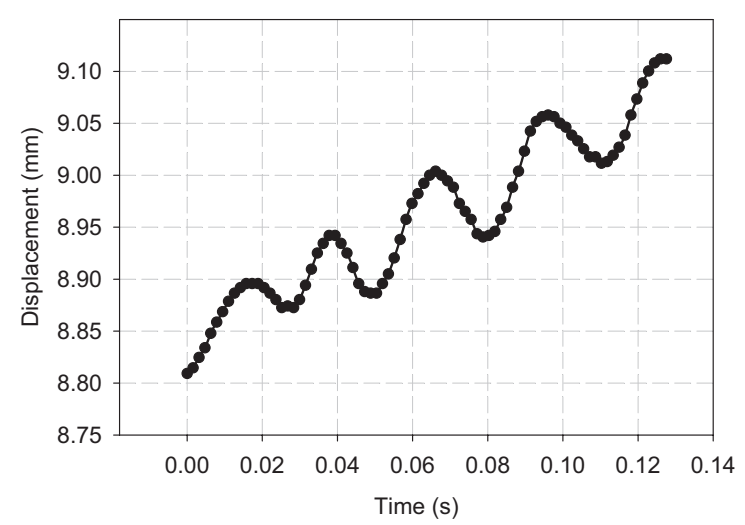

Figure 8: Position of the slider, X(t), in 130-ms time window captured at $635 \mathrm{fps}$ shows an average speed of $1.8 \mathrm{~mm} / \mathrm{s}$ at $10 \mathrm{~Hz}$ excitation, as well as a sinusoidal oscillation.

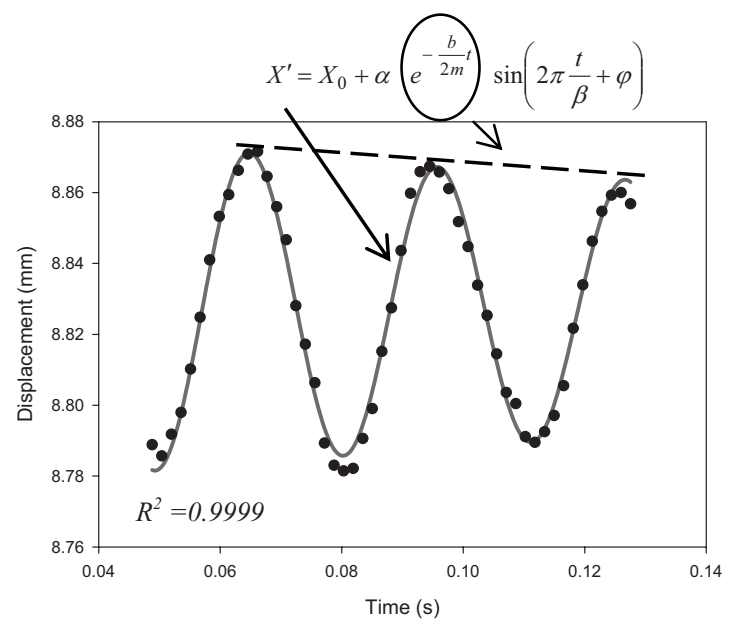

Figure 9: Local rotor displacement, $\left(X(t)-\left(V_{\text {avg }} \times t\right)\right)$, showing damped sinusoidal transient response. The standard coefficient of determination $\left(R^{2}\right)$, describing how well the data points are fitted to the curve, was calculated to be 0.9999 . 


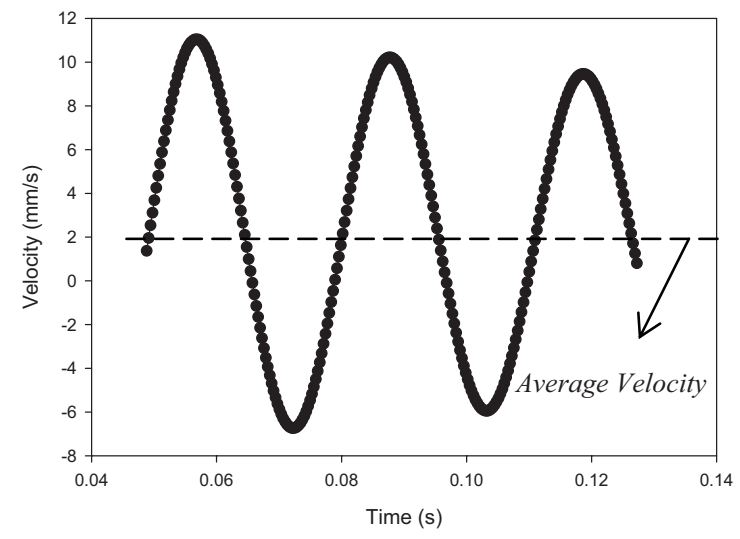

Figure 10: Instantaneous velocity of the slider with damped sinusoidal form was computed from the curve fit to the position data (Figure 8-9).

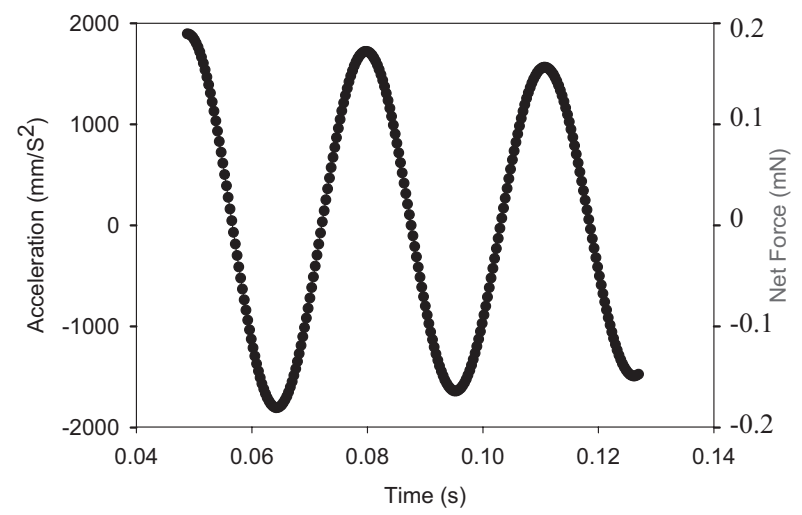

Figure 11: Instantaneous acceleration and the net force of the slider with damped sinusoidal form were computed from the velocity data shown in Figure 10.

\section{DISCUSSION}

The results from the position measurement using a 30-fps camera, summarized in Table 2, show a good agreement between predicted and measured values of the average velocity for the excitation frequency of $f_{e} \leq 60 \mathrm{~Hz}$. The motor does not pull into synchronization at $80 \mathrm{~Hz}$ or higher. This is due to a limited range of the linear micromotor $(3.4 \mathrm{~mm})$ and the change in the direction of motion. The micromotor does not have enough time to reach the synchronous speed before it reaches the end of the trench.

The period of the oscillations in the transient response measurements (Figures 7-11) was found to be independent of the frequency of excitation voltage. It is believed that this period is the result of the mechanical properties of the slider and the microball bearings support mechanism e.g. mass and damping. The measured net force, shown in Figure 11, is the result of the electromechanical force, produced by the machine, and the frictional force between microball bearings and the silicon housing.

\section{CONCLUSION}

The dynamic characterization of a bottom-drive, linear, variable-capacitance micromotor supported on microball bearings was presented. The slider position of the fabricated micromotor was measured using low- and high-speed video cameras; the instantaneous and average velocity, acceleration, net force, and the dashpot coefficient were extracted from the position measurement and are in good agreement with predicted values.

The characterization methodology presented in this paper is applicable to a wide range of micromachines and provides useful information for design, control, and understanding the dynamic behavior of variable-capacitance micromachines.

\section{ACKNOWLEDGMENTS}

This work supported by the Army Research Office through MURI Program under Grant No. ARMY-W911NF0410176, with Dr. Tom Doligalski as the technical monitor, the Army Research Lab under Grant No. CA\#W911NF-05-2-0026, and the National Science Foundation under Grant No. ECS-0224361. The authors would also like to thank Nitta Haas Company, Japan for providing the CMP supplies.

\section{REFERENCES}

[1] A. Modafe, N. Ghalichechian, J. H. Lang, and R. Ghodssi, "A Microball-Bearing-Supported Linear Electrostatic Micromotor with Benzocyclobutene Polymer Insulating Layers," TRANSDUCERS '05, pp. 693-696, Seoul, Korea, 2005.

[2] A. Modafe, N. Ghalichechian, A. Frey, J. H. Lang, and R. Ghodssi, "Microball-Bearing-Supported Electrostatic Micromachines with Polymer Dielectric Films for Electromechanical Power Conversion," Power MEMS '05, pp. 173-176, Tokyo, Japan, 2005.

[3] T. W. Lin, A. Modafe, B. Shapiro, and R. Ghodssi, "Characterization of dynamic friction in MEMS-based microball bearings," IEEE transactions on Instrumentation and Measurement, vol. 53, pp. 839-846, 2004.

[4] K. Deng, W. H. Ko, and G. M. Michal, "A preliminary study on friction measurements in MEMS," TRANSDUCERS '91, pp. 213-218, San Francisco, CA, USA, 1991.

[5] K. Noguchi, H. Fujita, M. Suzuki, and N. Yoshimura, "The measurements of friction on micromechatronics elements," MEMS '91, pp. 148-153, Nara, Japan, 1991.

[6] A. Modafe, N. Ghalichechian, B. Kleber, and R. Ghodssi, "Electrical characterization of Benzocyclobutene polymers for electric micro machines," IEEE Transactions on Device and Materials Reliability, vol. 4, pp. 495-508, 2004.

[7] N. Ghalichechian, A. Modafe, R. Ghodssi, P. Lazzeri, V. Micheli, and M. Anderle, "Integration of Benzocyclobutene Polymers and Silicon Micromachined Structures Using Anisotropic Wet Etching," Journal of Vacuum Science \& Technology B, vol. 22, pp. 2439-244, 2004.

[8] A. Modafe, N. Ghalichechian, M. Powers, M. Khbeis, and R. Ghodssi, "Embedded benzocyclobutene in silicon: An integrated fabrication process for electrical and thermal isolation in MEMS," Microelectronic Engineering, vol. 82, pp. 154, 2005.

[9] M. Mehregany, S. F. Bart, L. S. Tavrow, J. H. Lang, and S. D. Senturia, "Principles in design and microfabrication of variable-capacitance side-drive motors," Journal of Vacuum Science \& Technology A: Vacuum, Surfaces, and Films, vol. 8, pp. 3614-3624, 1990.

[10] S. F. Bart, M. Mehregany, L. S. Tavrow, J. H. Lang, and S. D. Senturia, "Electric micromotor dynamics," IEEE Transactions on Electron Devices, vol. 39, pp. 566, 1992. 